Int. J. Dev. Biol. 61: 537-543 (2017)

doi: $10.1387 /$ ijdb.170194oo

\title{
An empirical model of Onecut binding activity at the sea urchin SM50 C-element gene regulatory region
}

\author{
OCHAN OTIM* \\ Division of Biology, California Institute of Technology, Pasadena, CA, USA
}

\begin{abstract}
Studying the formation of endoskeleton in many species is complex and difficult. The sea urchin embryo offers an unparalleled platform for understanding this process because of the ease with which its skeletogenic mesenchyme cells can be manipulated. In this study, preliminary evidence from biochemical studies towards understanding the role of the Onecut transcription factor during sea urchin skeletogenic mesenchyme cell specification is presented. Based on the evidence, an empirical model is proposed showing how Onecut, together with associated co-factors, may be using the C-element of the SM50 gene regulatory region in advance of the sea urchin Strongylocentrotus purpuratus spicule development. In the model, Onecut recognizes and binds the DNA sequence CATCGATCTC in the $\mathrm{C}$-element without temporal restriction. Onecut then utilizes different sets of co-factors to switch from its unknown function early in development (four cell stage to the mesenchyme blastula stage), to its known role in the oral-aboral boundary thereafter. At the writing of this report, definitive evidence as to whether the "early" factors are expressed in all cells except the micromere lineages, or whether the "late" factors are expressed in micromere descendants or ectodermal precursors only are lacking. The former would suggest a possible Onecut repression function for the early co-factors outside the micromere lineages; the latter scenario would suggest a Onecut activation function for the late co-factors in the presumptive ciliary band.
\end{abstract}

KEY WORDS: C-element, HNF6, Onecut, sea urchin, SM50

\section{Introduction}

Studying the formation of endoskeleton in many species is a difficult process because of the complex nature of tissues involved. The purple sea urchin (Strongylocentrotus purpuratus, S. purpuratus), with its elaborate endoskeleton formation, has been playing a central role in advancing knowledge in this area because of the ease with which the primary mesenchyme cells (PMC) can be manipulated micro-surgically (Ettensohn and McClay 1988, and references therein), or separated and cultured in vitrountil deposition of mineralized tissue (Okazaki 1975; Lee et al., 1999). The PMC is a group of 32 cells that synthesize the sea urchin endoskeleton (see Lyon et al., 2014, and Minokawa 2016 for recent descriptions of the role of PMC during sea urchin development). Central to embryonic skeletal development studies in sea urchin are several cloned genes (Peled-Kamar et al., 2002; Sucov et al., 1987; Wilt 1999), but whose gene regulatory networks are yet to be completely dissected. The regulation of one such gene, the SM50, is of interest to us. SM50 encodes a differentiation product utilized exclusively in the skeletogenic lineages. That the expression of SM50 is spatially restricted during development was demonstrated by Sucov et al., (1988) who used a fusion construct SM50.CAT to show accurate spatial embryonic expression in the PMC. The SM50.CAT fusion construct included a genomic sequence from -440 to +120 with respect to the transcription start site. This genomic sequence was later shown to contain four adjacent regulatory elements, three of which are positively acting, and one, the C-element positioned downstream of the transcription start site and the subject of this study, is an indispensable spatial control element (Makabe et al., 1995). Another S. purpuratus gene which has also been shown to contain the $\mathrm{C}$-element in its putative regulatory region is the SM37 gene (Lee et al., 1999). Compared to the SM50 C-element, the SM37C-element has four base mismatches and one deletion. The role of C-element in SM37 regulation is not defined as yet. Within the broader Strongylocentrotidae family, the C-element is conserved among five of the eight closely related species (Walters et al., 2008).

Abbreviations used in this paper: EMSA, electrophoretic mobility shift assay; PMC, primary mesenchyme cell.

\footnotetext{
*Address correspondence to: Ochan Otim. Environmental Monitoring Division, City of Los Angeles, 12000 Vista Del Mar, Playa Del Rey, CA 90293, USA. Tel: +1 (310) 648-5835. Fax: +1 (310) 648-5828. E-mail: ochan.otim@lacity.org (ID https://orcid.org/0000-0001-7272-4356
} 
One sea urchin gene product which we believed has a role in regulating SM50 is Onecut (also known as HNF6), a factor of the cut-domain family of homeodomain proteins. In mammalian cells, Onecut is enriched in the liver, where it stimulates transcription of liver-expressed genes, and antagonizes glucocorticoid-stimulated gene transcription (Rausa et al., 2003). Recently, Onecut protein was shown to recognize the -5/-6 region of the human F9 promoter (5'-AACTAATCGACCTTACCA-3', Funnell et al., 2013), a sequence that includes an ATCGA motif in the Sucov et al., (1988) SM50. $C A T$ fusion construct.

In a preliminary study using recombinant Onecut expressed in bacteria, we showed that the factor binds specifically to the C-element (Fig. 10 in Otim et al., 2004) and, therefore, should be involved in embryonic endoskeleton formation. On that basis, we expected Onecut to regulate SM50 specifically in the skeletogenic cells or the PMC. However, attempt to test this hypothesis was sidetracked by the discovery of the spectacular role this factor plays in the development of the ciliated band, the oral-aboral boundary marker. Out of curiosity, we also found by whole-mount in situ hybridization that Onecut plays a similar role in marking the oralaboral boundary in a sea star Asterina miniata even though sea star and sea urchin diverged from a common ancestor around 500 million years ago (Otim et al., 2005). Knockdown of Onecut in sea urchin led to multiple consequences on embryonic development. This included failure to form fully developed spicules, the abnormal growth of a perfectly spherical embryo, the uncharacteristic formation of a centralized archenteron which fails to bend and fuse with the oral ectoderm (presumably because the oral ectoderm is absent in the knockdown), and the proliferation of pigment cells symmetrically arranges around the archenteron (Fig. 7 in Otim et al., 2004).

In this study (cited in its preliminary stage by Walters et al., 2008), biochemical tools including extensive electrophoresis mobility shift assay (EMSA, Hellman and Fried 2007) are employed in an attempt to assess, sort, and define empirically Onecut binding activities at the C-element sequence of SM50 regulatory region in $S$. purpuratus. Results reported should inform subsequent effort to understand the contribution of Onecut to the accurate expression of SM50 in the PMC or the skeletogenic lineages. Such effort may involve identifying and functionally analyzing all the direct and indirect Onecut-associated C-element binding proteins identified in this work.

\section{Results}

\section{Onecut is present in nuclear extracts and binds $C$-element DNA sequence specifically}

Classic EMSA was used to definitively show that in a 20-h old embryonic nuclear extract, factors that bind to the C-element DNA sequence found in the regulatory region of SM50 are present. The results confirm the presence of such proteins that can form specific complexes (indicated by three arrowheads in Fig. 1, lanes 2-4, 7-8) with the wild-type C-element probe (Labeled C-element probe in Fig. 1). The complexes were lost with an excess amount of unlabeled C-element competitor (Fig. 1, lanes 5, 6) and were supershifted with Onecut-specific antibodies (lanes 4, 8), but not affected by the presence of preimmune antibodies (Fig. 1, lane 3) or unlabeled SpP3A2 probe (Fig. 1, lanes 7-8). The SpP3A2 target site is unrelated to the $S M 50$ C-element. The preliminary
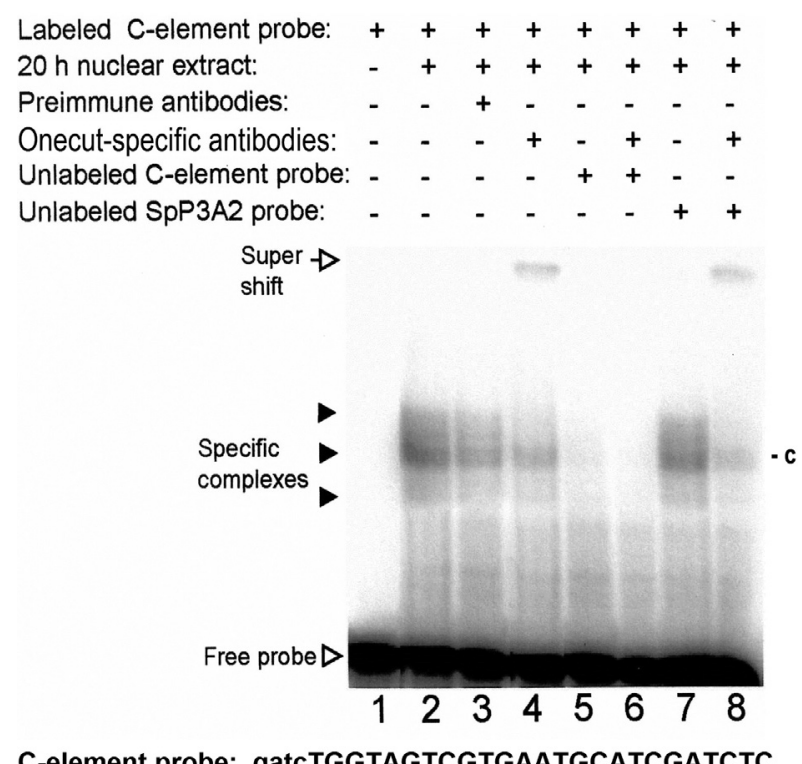

Fig. 1. Specificity test of Onecut-SM50 C-element binding by electrophoretic mobility shift assays (EMSA). EMSA of a 20-h Strongylocentrotus purpuratus embryonic nuclear proteins extract with the SM50 ${ }^{32}$ P-labeled C-element gatcTGGTAGTCGTGAATGCATCGATCTC oligonucleotide (lanes 2-8), rabbit pre-immune serum antibodies (lane 3), Onecut-specific rabbit antibodies (lanes 4,6,8), unlabeled C-element probe (lane 5,6) and unlabeled S. purpuratus $P 3 A 2$ probe (lane 7-8). Each binding reaction contained nuclear extract $(2 \mu \mathrm{L})$, poly (dl-dC)/poly(dl-dC) (200 ng) and ${ }^{32} \mathrm{P}$ end-labeled probe $(0.4 \mathrm{ng})$. The reactions were incubated on ice for $10 \mathrm{~min}$ prior to electrophoresis. The complex labeled "c" contains Onecut.

conclusion from these experiments is that the observed DNAprotein complexes (labeled $c$ ) contain Onecut as the major protein bound to the $\mathrm{C}$-element specifically. Note the reduction of the band intensities by adding antibodies to Onecut in Fig. 1 which occurs in all the bands referred to as "specific complexes" (compare lanes 4 and 8 with lanes 2 and 7 , respectively).

\section{Onecut binding on the $C$-element is limited to the sequence CATCGATCTC}

In traditional EMSA, the ${ }^{32} \mathrm{P}$-labeling of wild type probe is preferred. In this particular EMSA experiments however, ${ }^{32} \mathrm{P}$-labeled mutants $\mathrm{C} 2-\mathrm{C} 6$ competing with limiting amounts of unlabeled Celement wild type probe were preferred to accomplish two goals: to see if the mutations introduced specific binding sites, and to determine the exact target site on the C-element sequence of Onecut. EMSA was carried out with 20 -h nuclear extract. The use of limiting amounts of the wild type probe unambiguously optimized signal differences between DNA binding site not damaged by mutation and when only the wild type probe contains the intact binding site. The results presented in Fig. 2 show the formation of specific DNA-protein complexes (indicated by three arrowheads) with three of five transversely mutated C-elements C2, C3 and C4 when compared to $w$ t, the wild type (Fig. 2A, lanes 1-4). The DNA-protein complexes were lost when ${ }^{32} \mathrm{P}$-labeled mutants $\mathrm{C} 5$ and C6 were used (Fig. 2A, lanes 5-6) implying that the unlabeled wild type probe effectively outcompeted mutants $\mathrm{C} 5$ and $\mathrm{C} 6$ because these two mutant sequences are not recognized by the specific DNA binding proteins in the 20 -h nuclear extract. (Indeed, it ap- 
pears more bands than the major ones indicated by arrowhead are affected by $\mathrm{C} 5$ and $\mathrm{C} 6$ mutations.) These results indicate (i) the absolute requirement of the 10 bases CATCGATCTC (+28 to +37 with respect to transcription start site) for Onecut binding activities at the C-element, and (ii) that more than one protein is involved in this binding. The indispensable sequence CATCGATCTC includes a 6-base palindromic sequence ATCGAT as expected for a DNA binding site used by regulatory protein (Wyrwicz et al., 2007, Lis and Walther 2016), but with zero intervening length in the S. purpuratus SM50 C-element.

All the DNA-protein complexes are supershifted by Onecut specific antibodies except for C5 and C6 mutants (Fig. 2B) confirming not only the specificity of this complexes to Onecut protein, but that Onecut (with co-factors in tow) binds the DNA sequence

A

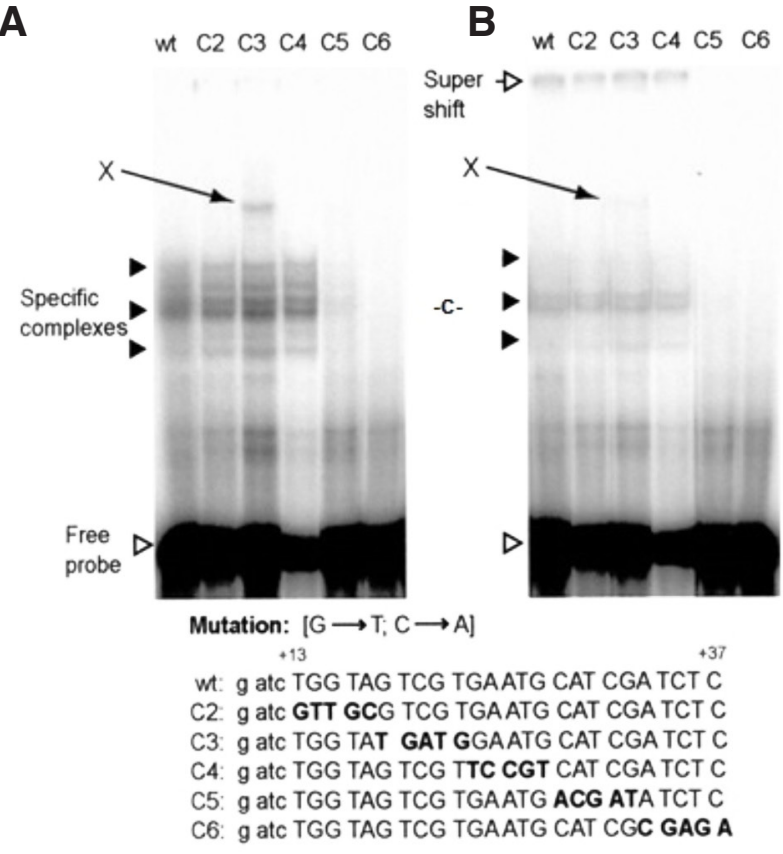

Onecut core binding site: CAT CGA TCT C

C

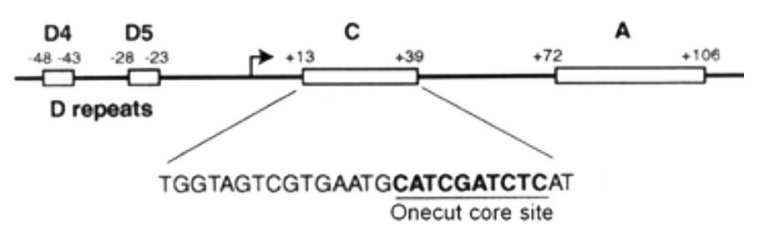

CATCGATCTC directly. The relative location of the indispensable 10 bases spanning $\mathrm{C} 5$ and $\mathrm{C} 6$ within the $\mathrm{C}$-element is shown in Fig. 2C together with the three adjacent positively acting regulatory elements identified by Makabe et al., (1995): two D-elements upstream and one A-element downstream of the transcription start site. Mutation represented by $\mathrm{C} 3$ introduces a non-SM50 sequence recognized specifically by a factor labeled $X$. The activity of $X$ would not have been visible had we used ${ }^{32} \mathrm{P}$-labeled wild type probe.

\section{Onecut forms unique temporal DNA-protein complexes during development}

To acquire precursory information on contributors to the DNAprotein complexes formed with the participation of Onecut in sea urchin development, EMSA was carried out using nuclear extracts prepared at an interval of about $3 \mathrm{~h}$ over the first $26 \mathrm{~h}$ after fertilization (Fig. 3). The EMSA temporal profile reveals at least eight unique DNA-protein complexes (labeled $a-h$ ). Complexes $b, d-g$ are clearly formed by early acting factors, and $h$ by late factors. Complex $a$ is observed transiently around 9 to $18 \mathrm{~h}$. The complex labeled $c$ contains Onecut (evidently supershifted by Onecut specific antibodies, Figs. 1-2) and is temporally unrestricted. The

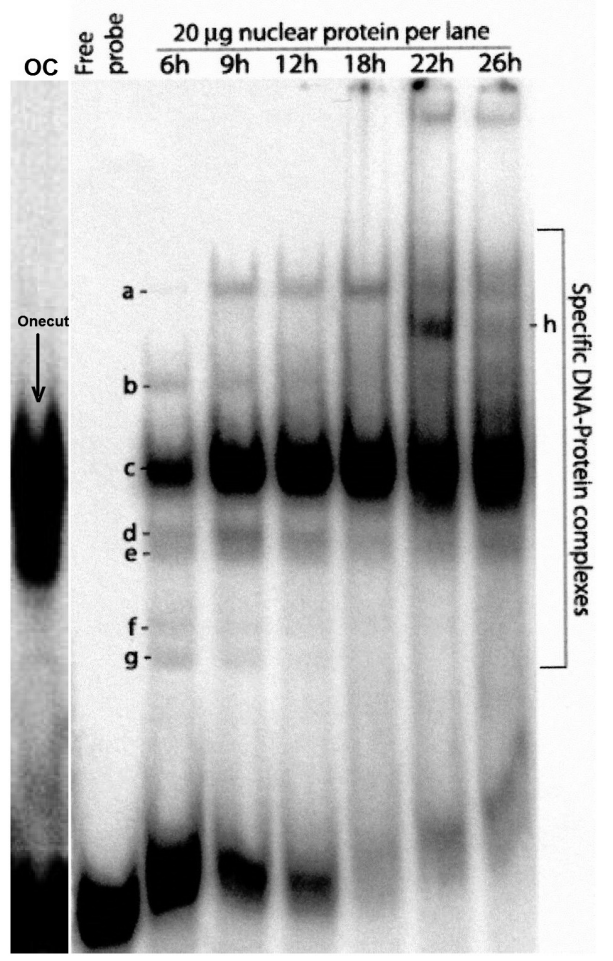

Fig. 2 (left). Determining Onecut target within the C-element. The C-element contains an indispensable Onecut target site comprising 10 bases as revealed by EMSA using transversion mutations shown below in bold face (G to $\mathrm{T}$, and $\mathrm{C}$ to $\mathrm{A}$ ). In lane 1, the control experiment, the wild type probe (wt) is ${ }^{32}$ P-labeled. In lanes 2-6, the mutants C2-C6 were ${ }^{32}$ P-labeled instead of, and competing with, unlabeled wild type probe for binding protein in the 20-h nuclear extract. DNA-protein complexes (also labeled c) are indicated by three arrowheads. (A) No Onecut specific antibody added; (B) DNAprotein complexes supershifted by binding to Onecut specific antibodies. Note that specific and supershift complexes are not observed with C5 and C6 mutants because the unlabeled wild type effectively outcompeted the mutants C5 and C6. These mutants are not recognized by the DNA binding proteins in the 20-h nuclear extract. (C) Makabe et al., (1995) cis-regulatory control elements of the SM50 gene showing the indispensable 10 bases found in this study within the C-element to be required for Onecut binding.

Fig. 3 (right). Temporal profile of the C-element binding proteins over the first $\mathbf{2 6} \mathbf{h}$ of embryonic development as revealed by EMSA. Embryonic nuclear proteins extracts were harvested from a vat of synchronously developing embryos at different stages of development (shown above) were incubated for $10 \mathrm{~min}$ with SM50 ${ }^{32}$ P-labeled C-element oligonucleotide in the presence of $200 \mathrm{ng}$ poly(dl-dC)/poly(dl-dC) and analyzed by electrophoresis. Lane "OC" represents an experiment with in vitro Onecut (see Otim et al., 2004 for details) and is included to identify complex containing Onecut, c. 
conclusion is that Onecut is constitutively present in all extracts and is associated collectively with at least eight factors within the developmental time frame of this study.

\section{Model of Onecut interaction at the C-element}

To determine the composition of the two distinct multimeric Onecut specific complexes detected early and late in development (Fig. 3), EMSA was carried out with nuclear extracts prepared from the four cells developmental stage embryos (6 $\mathrm{h}$ post-fertilization, Fig. 4A) and the blastula stage embryos (22 h post-fertilization, Fig. 4B) using limited amounts of (and this time) ${ }^{32} \mathrm{P}$-lebeled wild-type $\mathrm{C}$-element competing with excess unlabeled mutants $\mathrm{C} 2-\mathrm{C} 6$. This reversed labeling vis-a-vis experiments in Fig. 2 was to allow contrast between these experiments and those presented in Fig. 2. Results show that in both the four cell stage and the blastula stage, the mutants C2, C3 and C4 outcompeted the wild-type for the Onecut major complex formation (indicated by $c$ and the oval shape in Fig. 4, and identified by gel supershift assay in Figs. 1 and 2) confirming that the Onecut binding site is not affected by mutations C2, C3 and C4 of the C-element. The two adjacent mutations at C5 and C6 covering 10 bases render the unlabeled mutants uncompetitive for the Onecut associated complex. Although few in numbers in these experiments, the ${ }^{32} \mathrm{P}$ labeled wild type probe was able to bind effectively to the Onecut associated proteins in the nuclear extracts at the expense of the mutants. These results confirm the importance of the stretch of

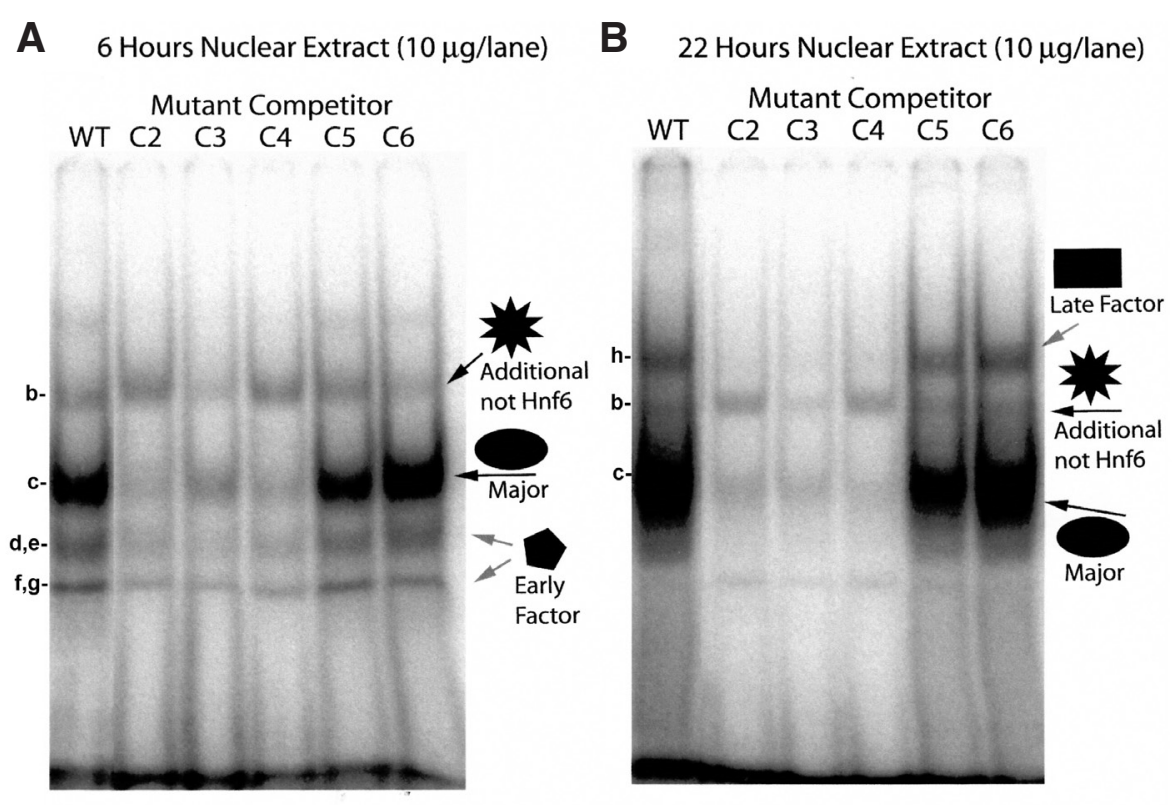

C

Model of Interaction at the C-Element

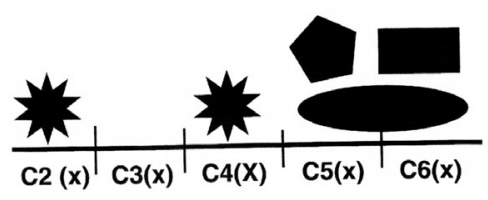

Fig. 4. Composition of Onecut binding activities at the SM50 C-element. Determining the composition of, and comparing the SM50 C-element binding activities early (A) and late (B) in development by competitive EMSA using a 1:1

mixture of unlabeled mutants (Fig. 2) and ${ }^{32}$ P-labeled wild-type C-element in all lanes (unlike experiments in Fig. 2 where the mutants are ${ }^{32}$ P-labeled in lanes 2-6). Other conditions are as described in Fig. 1. (C) An empirical model summarizing our interpretation of Onecut binding activity at the sea urchin SM50 C-element gene regulatory region as revealed by EMSA.
DNA sequence CATCGATCTC to Onecut binding.

Also shown in Fig. 4 as two 10-point star polygons (labeled "Additional not Onecut" and $b$ ) are factors that bind the 25 base pair study sequence in a way distinctively different from the pattern shown by Onecut. The signal strength of these DNA-protein complexes appear to suggest that these additional factors are (heir binding activities are weak and not affected these results; that these additional factors are not competing with Onecut for the same site. Mutation C3 appears to reduce binding (hese factors but enhances the presumptive Onecut 3 , the $b$ factors are attached to Onecut. We also believe that wild type probe competitive binding, but the Onecut associated (oval, Fig. 4). bands $d-g$ indicated by arrov mirror proportionally that of Onecut in that both sets of signals are diminished by mutations at C2, C3 and C4. This may suggests that the two factors (early and late) are trans-acting and are directly ssociated with Onecut.

In Fig. 4C is shown an integrated proposal summarizing all the interactions discussed at the two embryonic developmental stages (the four cell and the blastula stages, Figs. 4A and 4B, respectively). The constitutively present factors labeled collectively as $b$ are bound at $\mathrm{C} 2$ and $\mathrm{C} 4$; Onecut with its two trans-acting switching factors $(d-g$ and $h$ ) are bound to the DNA sequence stretch whose mutations are labeled C5 and C6.

\section{Onecut binding activity is ionic strength dependent}

To glean into the stoichiometry of binding at the C-element and, particularly, to determine whether Onecut binding depends on ionic strength, a 20-h nuclear extract was passed through a tandem of C-element oligonucleotide affinity columns, washed extensively and eluted in steps with solutions containing increasing amounts of $\mathrm{KCl}$ as shown on top of Fig. 5A. Ten fractions were collected and the specific DNA-protein binding activities were monitored in each fraction by EMSA on a $10 \%$ native polyacrylamide gel; other EMSA conditions are as described in Fig. 1 caption. The EMSA profile of the ten fractions were compared with that of an experiment performed using unpurified 20-h nuclear extract (lane C, Fig. 5A) to identify the specific complexes $c$ and $h$ present in the late embryonic stages (Fig. 3). The affinity purified wild-type Onecut protein was identified by Southwestern blot analysis (Fig. 5B) and its molecular mass was determined to be about $52 \mathrm{kDa}$ as expected (the size of 
A

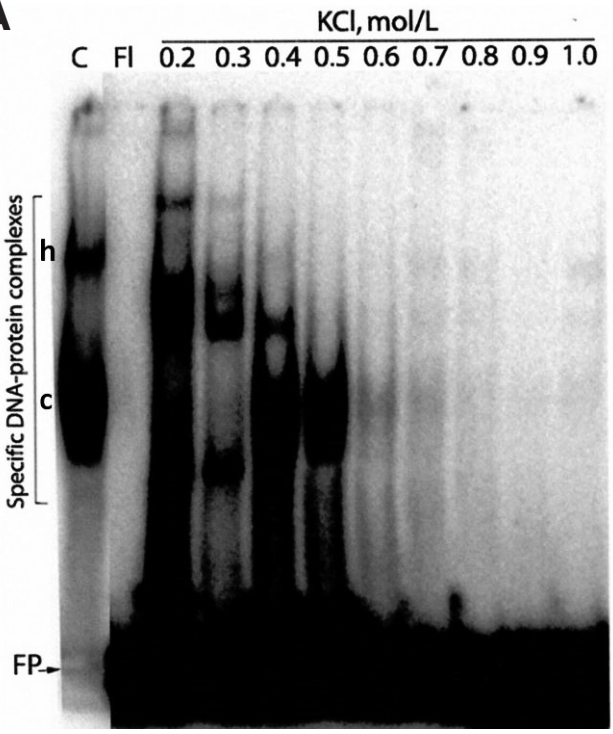

B

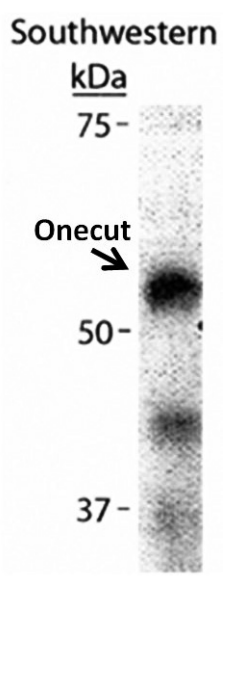

Fig. 5. Purification of native Onecut proteins. (A) Partial purification of Onecut binding proteins (late acting factors) from a 20-h embryonic nuclear extract by affinity chromatography. The sequence of the concatenated oligonucleotide used for affinity chromatography was 5'gatcTGGTAGTCGTGAATGCATCGATCTC (probe) corresponding to +13 to +37 region from the transcription start site of Onecut (Fig. $2 \mathrm{C}$ without the last AT). C, FI and FP refer to unpurified binding reaction used here as negative control of elution, flow-through column blank and free probe as the positive control, respectively. The nuclear extract was passed through a tandem of oligonucleotide affinity columns, washed extensively and eluted in steps with solutions containing increasing amounts of $\mathrm{KCl}$ as shown on top. Ten fractions were collected and the specific DNA-protein binding activities were monitored in each fraction by mobility shift assay on a 10\% native polyacrylamide gel as described in Fig. 1 caption. At the minimum, $\mathrm{C}$-element binding factors $\mathrm{c}$ and $\mathrm{h}$ (Fig. 3) are present in the $20 \mathrm{~h}$ nuclear extracts (no salt, lane $C$ ). Notice that the complex hardest to disrupt is the Onecut-DNA complex c (c is missing in $0.3 \mathrm{~mol} / \mathrm{L} \mathrm{KCl} \mathrm{eluent);} \mathrm{h}$ appears to be loosely bound and is completely eluted at $0.3 \mathrm{~mol} / \mathrm{L} \mathrm{KCl}$. (B) Identifying affinity purified transcription factors by Southwestern blot analysis. Onecut is shown by the arrow (molecular mass $52 \mathrm{kDa}$ ).

full length Onecut synthesized from cDNA clone 195A17 (Otim et al.2004) is $52 \mathrm{kDa}$ ). It is clear from Fig. $5 \mathrm{~A}$ that increasing $\mathrm{KCl}$ concentration disrupts rather than augments the binding of Onecut protein to DNA. This finding is outside recent discoveries that binding of some transcription factors to DNA displays virtually zero dependence on ionic strength under these conditions (Mikles et al., 2014). The most stable DNA-protein complex to disruption is $c$, that which contains Onecut. Indeed, the DNA motif utilized by Onecut is known to form a stable DNA-protein complex (Nepveu 2001). The C-element binding factor $h$ found in the 22-h nuclear extract shown in Fig. 3 is present as well in the 20 -h nuclear extract used here (Fig. 5A); factor $h$ appears to be loosely bound and is completely eluted at $0.3 \mathrm{~mol} / \mathrm{L} \mathrm{KCl}$. Note that no band is present at the position corresponding to band $c$ in the $0.3 \mathrm{~mol} / \mathrm{L} \mathrm{KCl}$ eluent; this is an anomaly. Note also that only $h$ and $c$ bands in the control experiment (no salt, lane $\mathrm{C}$ ) can be labeled with certainty in Fig. 5A (and may be $c$ elsewhere) because of ambiguity in predicting the effect of salt on this multiplex DNA-protein associations. Additionally, the range of ionic strengths used in these experiments is not found in living sea urchin cells and, therefore, these experimental results cannot be used to discuss the function of Onecut as a transcription factor.

\section{Discussion}

\section{Structure of Onecut binding site and co-factors involved}

Transcriptional regulation results from a complex organization of cis-acting sequences that serve as binding sites for a multitude of trans-acting factors which together determine the activity of a gene. In higher eukaryotes, these cis-acting sequences are typically clustered into discrete functional modules. We found such clustering to be present in the C-element regulatory region of the SM50 gene. At least three short sequences labeled C2 (TGGTA), C3 (GTCGT), C4 (GAATG), and C5-C6 (CATCGATCTC) are clearly required for the regulatory control of this gene, the latter of which is specifically utilized by Onecut. Detected also were at least eight factors associated with the C-element; five of these interactions are preliminarily accounted for in Fig. 4 C.

\section{Early factor and late factor are potential determinants of Onecut function}

Based on data obtained, the function of "early factor" and "late factor" would be of interest to understanding the role of Onecut in the context of this work. Given that the nuclear extracts used in this study are a mixture of transcription factors expressed in different embryonic territories, the first question to ask would be whether these early and late factors are expressed in different portion of embryos (such as micromere descendants or ectodermal precursors). If so, these co-factors should be prime candidates for defining the role of the Onecut transcriptional complex. In one scenario, Onecut could be the portion responsible for DNA binding in the complex, while the co-factors define the specific function of the complex. The early factors could then function as transcription repressors of Onecut, and are expressed in all cells except the micromere lineages while the late factors functions as transcription activators, and are expressed in the presumptive ciliary band. In this arrangement, the early factors functionally compete with the late factors for Onecut binding which effectively means Onecut is neutral for transcriptional function without these co-factors. Such an explanation (that the DNA sequence CATCGATCTC within the C-element has no transcriptional activity on its own) would fit well with Makabe's hypothesis (Makabe et al., 1995). A similar relationship is the well-known Tcf, $\beta$-catenin, and groucho transcription system whereby the transcription factor Tcf is responsible for DNA binding, the cofactor $\beta$-catenin then mediates transcription activation in embryonic territory where the co-factor groucho level is low, and groucho mediates transcription repression in territory where $\beta$-catenin level is low (Range et al., 2005).

In an alternative scenario, Onecut may actually be using a very different regulatory element (not the $\mathrm{C}$-element) to regulate the formation of the presumptive ciliary band, hence linking the oralaboral axis formation to results describe here at this time would be premature.

The following conjecture could also be made about the function of Onecut even though the experiments described in this paper are inadequate. Of the four SM50 elements (A, C, D4 and D5, Fig. $2)$, the C-element has been shown to have two very specific roles: one of directing SM50 expression to the PMC, and the other whose mutation kills the ability of SM50 to express at all (Makabe et al., 
1995). Furthermore, it is known that the palindromic ATCGAT DNA sequence (shown in this study to bind Onecut, C5-C6 in Fig. 2) enables factors with the cut homeodomain (such as Onecut), to repress transcription by forming a stable repressor DNA-protein complex (Nepveu, A. 2001). It then becomes impossible to rule out Onecut transcription complex from being part of a repression mechanism of SM50 expression elsewhere in a developing embryo but the PMC or the skeletogenic lineages. This is supported by previous finding that (i) Onecut mRNA is present everywhere in the embryo until about $30 \mathrm{~h}$ after fertilization when expression is localized in the region where the ciliated band would arise (see Fig. 6 in Otim et al., 2004; hnf6 is Onecut) and that (ii) no correlation was observed between the expression pattern of SM50 and Onecut $24 \mathrm{~h}$ after fertilization (see Fig. 11 in Otim et al., 2004). This means the repressive role of Onecut complex in this context is not required everywhere except where it is needed to regulate the development of the ciliated band after the sea urchin's endoskeleton has been assigned to the right address.

\section{Concluding remarks}

We have shown by EMSA that proteins in a 6-h and a $20-\mathrm{h}$ sea urchin embryonic nuclear extract bind the C-element of the SM50 gene specifically, and that one complex is the major (strongest ${ }^{32} \mathrm{P}$ signal) and the most tightly bound to DNA (requiring high concentrations of $\mathrm{KCl}$ to disrupt). We have also demonstrated that the major DNA-protein complex is supershifted by antibodies raised against Onecut implying a protein in the major complex is Onecut. This was confirmed by both western and southwestern blots. Through mutational studies, the exact DNA region within the $\mathrm{C}$-element recognized by Onecut protein was found to be this short sequence: CATCGATCTC. A five time-point EMSA "titration" after fertilization suggests that Onecut utilizes different co-factors as control switch at different regulatory stages of development. The detail of this complexity was investigated at the four cell stage and at the blastula stage of embryonic development by competitive EMSA using a 6-h and a 22-h sea urchin embryonic nuclear extract, respectively. Demonstratively, a distinct and low level collection of early factors, and of late factors appear to be but unaffected by mutations. A model of interactions at the C-element is proposed. Still needed though to firmly support the proposed model, a largely untested hypothesis, are experiments revealing the identity, function and expression of the putative auxiliary factors.

\section{Materials and Methods}

\section{Expression and purification of Onecut bacterial protein}

The cDNA clone 224C2 (Fig. 1A, Otim et al., 2004) was double digested with EcoRI (New England Biolabs, Ipswich, MA, USA) at an internal site $160 \mathrm{bp}$ from the 5' end of the clone, and with HindlII (New England Biolabs, Ipswich, MA, USA) at a site that adds six amino acids (34 bp containing a stop codon) from a plasmid vector pSPORT (Invitrogen, Carlsbad, CA, USA) that was used to construct a 20-h cDNA library. The digest was cloned in frame into the Hindll and EcoRI sites of the pRSETC expression vector (Invitrogen, Carlsbad, CA, USA) and expressed to produce a $39 \mathrm{kD}$ recombinant SpCut224 fusion protein. Antibodies were raised against this fusion protein, the specificity of which was tested by western blots using nuclear extracts prepare from 6-h, 12-h, 24-h, 48-h, and 72-h embryonic developmental stages (Otim unpublished), and by southwestern blot.

\section{Electrophoresis mobility shift assay}

The synthetic SM50 C-element wild type (wt) sequence used as EMSA probes on the post-fertilization nuclear extracts was a double stranded DNA strand resulting from annealing a 1:1 mole ratio of sequences $S M 50 C+1$ : 5' gatcTGGTAGTCGTGAATGCATCGATCTC and SM50C-1: 5' gatcGAGATCGATGCATTCACGACTACCA. The sequence spans the region from +13 to +37 nucleotides from the transcription start site. Nucleotides in lower case are terminal tags for end labeling. For experiments involving the C-element mutants, the region to be mutated was transversionally changed (i.e. $\mathrm{G}$ to $\mathrm{T}$ and $\mathrm{C}$ to $\mathrm{A}$ ). Mutations were introduced in the form of synthetic oligonucleotides.

\section{Temporal scale definition}

In this report, early is arbitrarily defined as the first $6 \mathrm{~h}$ after fertilization; late as more than $20 \mathrm{~h}$ after fertilization. Hours between 6 and 20 are assumed to contain different shades of events at $6 \mathrm{~h}$ and $20 \mathrm{~h}$.

\section{Acknowledgments}

The author thanks Dr. Eric H. Davidson (EHD) of this institute for guidance and Dr. Chiou-Hwa Yuh, also of this institute, for unparalleled hands-on support. The author also thanks Dr. Takuya Minokawa of Tohoku University, Japan, and Dr. Gabriele Amore of the Istituto Regionale Vini E Oli, Italy, for critically reviewing this manuscript. This work was supported by NIH grant HD-37105 to EHD and by the Caltech Beckman Institute. The author has no industrial links or affiliations

\section{References}

COFFMAN J A and YUH C H (2004). Identification of Sequence-specific DNA Binding Proteins. Methods Cell Biol 74: 653-675.

ETTENSOHN C A and MCCLAY D R (1988). Cell lineage conversion in the sea urchin embryo. Dev Biol 125: 396-409.

FUNNELL A P, WILSON M D, BALLESTER B, MAK K S, BURDACH J, MAGAN N, PEARSON R C, LEMAIGRE F P, STOWELL K M, ODOM D T, FLICEK P and CROSSLEY M. (2013). A CpG Mutational hotspot in a ONECUT binding site accounts for the prevalent variant of Hemophilia B Leyden. Am J Hum Genet 92: 460-467.

HELLMAN L M and FRIED M G (2007). Electrophoretic mobility shift assay (EMSA) for detecting protein-nucleic acid interactions. Nat Protoc 2: 1849-1861.

LEE Y-H, BRITTEN R J and DAVIDSON E H (1999). SM37, a skeletogenic gene of the sea urchin embryo linked to the SM50 gene. Develop Growth \& Differ41: 303-312.

LIS M and WALTHER D (2016). The orientation of transcription factor binding site motifs in gene promoter regions: does it matter? BMC Genomics 17: 1-21.

LYONS D C, MARTIK M L, SAUNDERS L R and MCCLAY D R (2014). Specification to biomineralization: following a single cell type as it constructs a skeleton. Integr Comp Biol 54: 723-733.

MAKABE K W, KIRCHHAMER C V, BRITTEN R J and DAVIDSON E H (1995). Cisregulatory control of the $S M 50$ gene, an early marker of skeletogenic lineage specification in the sea urchin embryo. Development 121: 1957-1970.

MIKLES D C, BHAT V, SCHUCHARDT B J, MCDONALD C B and FAROOQA (2014). Enthalpic factors override the polyelectrolyte effect in the binding of EGR1 transcription factor to DNA. J Mol Recogni 27: 82-91.

MINOKAWA T (2016). Comparative studies on the skeletogenic mesenchyme of echinoids. Dev Biol 427: 212-218.

NEPVEUA(2001). Role of the multifunctional CDP/Cut/Cux homeodomain transcription factor in regulating differentiation, cell growth and development. Gene 270: 1-15.

OKAZAKI K (1975). Spicule formation by isolated micromeres of the sea urchin embryo. Amer Zool 15: 567-581.

OTIM O, AMORE G, MINOKAWA T, MCCLAY D R and DAVIDSON E H (2004). SpHnf6, a transcription factor that executes multiple functions in sea urchin embryogenesis. Dev Biol 273: 226-243.

OTIM O, HINMAN V F and DAVIDSON E H (2005). Expression of AmHNF6, a sea star orthologue of a transcription factor with multiple distinct roles in sea urchin 
development. Gene Expr Patterns 5: 381-386

PELED-KAMAR M, HAMILTON P and WILTF H(2002). Spicule matrix protein LSM34 is essential for biomineralization of the sea urchin spicule. Exp Cell Res272:56-61.

RAFIQ K, CHEERS M S and ETTENSOHN C A (2012). The genomic regulatory control of skeletal morphogenesis in the sea urchin. Development 139: 579-590.

RANGE R C, VENUTI J M and MCCLAY D R (2005). LvGroucho and nuclear betacatenin functionally compete for Tcf binding to influence activation of the endomesoderm gene regulatory network in the sea urchin embryo. Dev Biol279: 252-267.

RAUSAF M, TANY and COSTAR H (2003). Association between hepatocyte nuclear factor 6 (HNF-6) and FoxA2 DNA binding domains stimulates FoxA2 transcriptional activity but inhibits HNF-6 DNA binding. Mol Cell Biol 23: 437-449.

SUCOV H M, BENSON S, ROBINSON J J, BRITTEN R J, WILT F and DAVIDSON $\mathrm{E} H$ (1987). A lineage-specific gene encoding a major matrix protein of the sea- urchin embryo spicule.2. Structure of the gene and derived sequence of the protein. Dev Biol 120: 507-519.

SUCOV H M, HOUGH-EVANS B R, FRANKS R R, BRITTEN R J and DAVIDSON E $H$ (1988). A regulatory domain that directs lineage-specific expression of a skeleta matrix protein in the sea urchin embryo. Genes Dev 2: 1238-1250.

WALTERS J, BINKLEY E, HAYGOOD R, and ROMANO L A (2008). Evolutionary analysis of the cis-regulatory region of the spicule matrix gene SM50 in strongylocentrotid sea urchins. Dev Biol 315: 567-578.

WILT F H (1999). Matrix and mineral in the sea urchin larval skeleton. J. Struct. Biol 126: 216- 226.

WYRWICZ LS, GAJ P, HOFFMANN M, RYCHLEWSKI L and OSTROWSKI J (2007). Acommon cis-element in promoters of protein synthesis and cell cycle genes. Acta Biochim Pol 54: 89-98. 


\section{Further Related Reading, published previously in the Int. J. Dev. Biol.}

Role of Mad2 expression during the early development of the sea urchin

Odile Bronchain, Wael Jdey, Laetitia Caraty and Brigitte Ciapa

Int. J. Dev. Biol. (2017) 61: 451-457

https://doi.org/10.1387/ijdb.170053bc

Genome-wide identification of enhancer elements

Sarah Tulin, Julius C. Barsi, Carlo Bocconcelli and Joel Smith

Int. J. Dev. Biol. (2016) 60: 141-150

https://doi.org/10.1387/ijdb.160108jb

Brachyury, Tbx2/3 and sall expression during embryogenesis of the indirectly developing polychaete Hydroides elegans

Cesar Arenas-Mena

Int. J. Dev. Biol. (2013) 57: 73-83

https://doi.org/10.1387/ijdb.120056ca

\section{Genomic control of patterning}

Isabelle S. Peter and Eric H. Davidson

Int. J. Dev. Biol. (2009) 53: 707-716

https://doi.org/10.1387/ijdb.072495ip

\section{Developmental gene network analysis}

Roger Revilla-i-Domingo and Eric H Davidson

Int. J. Dev. Biol. (2003) 47: 695-703

http://www.intjdevbiol.com/web/paper/14756345

5 yr ISI Impact Factor $(2013)=2.879$
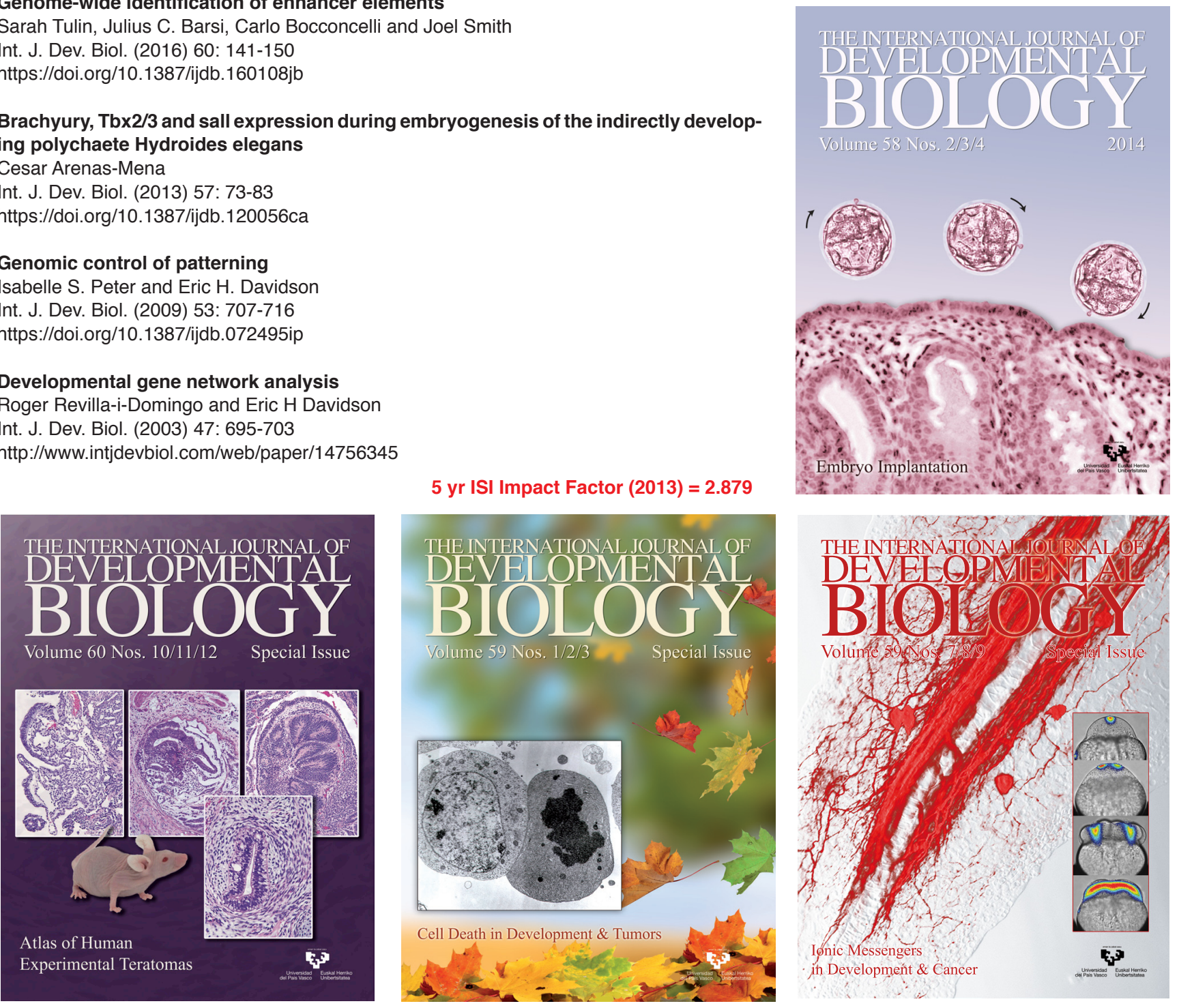